Article

\title{
Optimal Decision-Making to Charge Electric Vehicles in Heterogeneous Networks: Stackelberg Game Approach
}

\author{
Shijun Chen ${ }^{1}\left(\right.$, Huwei Chen ${ }^{2, *}$ and Shanhe Jiang ${ }^{1}$ \\ 1 School of Physics and Electronic Engineering, Anqing Normal University, Anqing 246133, China; \\ ch_sh_jun@sina.com (S.C.); jshxlxlw@163.com (S.J.) \\ 2 Department of Computer Science and Technology, Tsinghua University, Beijing 100084, China \\ * Correspondence: chenhuwei2008@163.com
}

Received: 20 November 2018; Accepted: 14 January 2019; Published: 21 January 2019

check for updates

\begin{abstract}
Electric vehicles (EVs) are designed to improve the efficiency of energy and prevent the environment from being polluted, when they are widely and reasonably used in the transport system. However, due to the feature of EV's batteries, the charging problem plays an important role in the application of EVs. Fortunately, with the help of advanced technologies, charging stations powered by smart grid operators (SGOs) can easily and conveniently solve the problems and supply charging service to EV users. In this paper, we consider that EVs will be charged by charging station operators (CSOs) in heterogeneous networks (Hetnet), through which they can exchange the information with each other. Considering the trading relationship among EV users, CSOs, and SGOs, we design their own utility functions in Hetnet, where the demand uncertainty is taken into account. In order to maximize the profits, we formulate this charging problem as a four-stage Stackelberg game, through which the optimal strategy is studied and analyzed. In the Stackelberg game model, we theoretically prove and discuss the existence and uniqueness of the Stackelberg equilibrium (SE). Using the proposed iterative algorithm, the optimal solution can be obtained in the optimization problem. The performance of the strategy is shown in the simulation results. It is shown that the simulation results confirm the efficiency of the model in Hetnet.
\end{abstract}

Keywords: electric vehicles; heterogeneous networks; demand uncertainty; power optimization; Stackelberg game

\section{Introduction}

Recently, electric vehicles (EVs) with low gas emission and environment protection have attracted much attention and have been widely applied in some countries when harsh environment problems are exposed in the world, such as energy shortage, air pollution, and the greenhouse effect [1-4]. Considering the increasing number of EVs, many more charging stations have been built to supply charging service. However, charging stations always have to confront the overload problem of charging EVs with a large amount of charging power demand. Then, EVs cannot be charged in time, which will restrict EV users' quality of experience (QoE) $[5,6]$.

Due to the limited capacity of batteries, EVs usually have to be charged with low SOC. Recently, charging stations have attracted much attentions and have been recognized as an available approach to supply charging service [7]. However, confronting the growing number of EV users, both waiting time and the power bill account for a major part of their payment. Though some different incentive mechanisms have been developed so far, there are still some problems, i.e., the optimal strategy of power management is usually designed based on the demand-side of EV users, which neglects the 
performance of wireless communication, e.g., wireless sensor networks (WSN) and vehicular-grid networks (V2G) [8].

With the development of communication technology, more and more wireless communication technology has been used in EVs and charging stations, e.g., vehicular ad-hoc networks (VANETs) and cognitive radio networks are applied to make the routing choice for EV users $[9,10]$. Different from the existing works, we study how to control the power supply among EV users, charging station operators (CSOs), and smart grid operators (SGOs) in the heterogeneous networks (Hetnet). Through Hetnet, there are many advantages for the charging system: (i) CSOs can exchange the real-time information with EV users, by which it helps EV users avoid the charging peak periods and reduce the expenditure of charging service. (ii) The power efficiency can be improved based on the optimal strategy to be designed in Hetnet, which can solve the problem of limited power supply. (iii) It can conveniently supply power to EV users and improve the profits of SGOs with less gas emission [11].

However, in order to improve the quality of experience (QoE), there are still some challenges to be improved and studied in the charging system when adopting the wireless communication [12,13]. These challenges include how to design the optimal strategy for CSOs, while benefiting both EV users and power retailers, respectively. At the same time, few works have been performed to make the optimal strategy in Hetnet [14-16]. Currently, many different power management approaches have been developed in the literature, aiming for scheduling the power supply with high efficiency [17-19]. In the existing works, the proposed approaches cannot directly be used in our paper, which have been designed without consideration of the performance of wireless communication.

In this paper, we propose the optimal incentive decision-making scheme to charge EVs in Hetnet. Firstly, the network model is designed in Hetnet, through which EV users can conveniently be supplied by CSOs. Then, based on the trading relationship among EV users, CSOs, and SGOs, we design the utility function for each of them in this charging system, respectively. In this function, the performance of Hetnet is integrated with the waiting time of EV users. Taking the bit error ratio (BER) in Hetnet, the load uncertainty is also analyzed and studied. In addition, in order to obtain the optimal strategy, we propose a four-stage Stackelberg game scheme. Namely, this charging power problem is formulated as an optimization problem. Through the back induction method, we get the Stackelberg equilibrium (SE), in which we also analyze and prove its existence. With less computation, we present an iterative search algorithm to obtain the optimal solution. Finally, simulation results demonstrate the effectiveness of our proposal.

The contributions of our paper are summarized as follows:

- In order to supply convenient charging service for EV users, the network model is designed. Through Hetnet, the information of each one in this charging system can be obtained. Here, as an important criterion in Hetnet, BER is taken into account, by which power loss is brought about.

- Based on the interaction among EV users, CSOs, and SGOs, we develop the utility function of each one in the charging system, respectively. Simultaneously, load uncertainty is studied and analyzed, by confronting BER in Hetnet. In order to schedule the power supplied from SGOs, we propose a four-stage Stackelberg game scheme. Then, the charging problem is formulated as an optimization problem. Through the theoretical analysis, we prove the existence and uniqueness of Stackelberg equilibrium (SE) in the proposed scheme.

- With less calculation, we present an iterative search algorithm to achieve SE with maximum profits of each in the charging system. At last, the simulation results verify the effectiveness of our proposed algorithm.

The rest of the paper is organized as follows. Section 2 presents a brief overview of the related work. In Section 3, we design the system model and reveal how to exchange the information in Hetnet. In Section 4, a Stackelberg game model is proposed to schedule the power supply. Meanwhile, an iterative search algorithm is presented to achieve optimal solutions. Both simulation results and related analysis are provided in Section 5, and then, the conclusions are given in Section 6. 


\section{Related Work}

Based on the development of wireless communication, both charging service for EV users and advanced communication technologies have been integrated. Through this approach, the QoE of EV users can be greatly improved with the efficiency of supplying power. Manshadi et al. in [20] studied how to operate the charging station based on the relationship between the electricity network and the transportation network. In order to supply the efficient and stable charging service to EV users, Sun et al. in [21] designed a novel software-defined framework. Yang et al. in [22] proposed a flexible real-time power management protocol for charging EVs based on the cooperation of each EV. In the process of managing power, the charging and discharging decision of each EV could be chosen in real time. Considering the charging problem based on the wireless sensor network, Zhang et al. in [23] formulated the wireless charging problem as an NP-hard scheduling problem. Hu et al. in [24] designed an energy management scheme for charging stations to utilize energy efficiently in the software-defined V2G network. However, the proposed algorithm in the above works mainly focused on how to schedule the power for EV users and power retailers, neglecting the power supply from the power grid. The performance of wireless communication is not taken into account, which is very important in scheduling the power for EV users.

Due to the performance of EV users' stochastic arrival and departure from charging stations, the queuing model is widely used to analyze this problem [25]. In order to solve the charging schedule, researchers studied many methods based on the different objectives. N. Liu et al. in [13] proposed a novel heuristic algorithm to schedule the power in the commercial building microgrid. The state of charge (SOC) of EV batteries was considered with the output of PV and the charging rate. W. Yuan et al. in [19] studied the charging strategy to maximize users' utility based on the two-stage Stackelberg model by using the queue model of $\mathcal{M} / \mathcal{G} / \mathcal{K}$, in which the location of charging facility and electrical price were discussed at the same time. I. Bayram et al. in [26] presented the pricing strategy to satisfy EV users' power demand based on the QoS, while shifting the power supply shortage during peak hours. M. Karbasioun et al. in [27] proposed the control policy with the minimal cost for the operator based on the real-time pricing scheme. C. Jin et al. in [28] investigated the charging schedule of the electric vehicles with the energy storage in the electricity market under the real-time price. M. Ismail et al. in [29] studied the profits of operators with queue theory and the proposed search algorithm to find the optimal outlets and capacity size in the charging facility. A. Ovalle et al. in [30] presented the charging schedule of EVs with the forward dynamic programming and game theory approach with the given constraints related to EVs. In addition, considering the driving routing of EV users with its corresponding constraints, artificial intelligence algorithms are usually used to decide the optimal strategy in power management [27]. In the existing works, they only considered that the dynamic event triggering affected the charging power, except the queue waiting time. Though the optimal solution could be obtained, they neglected users' different interests with the waiting space size [31,32].

In contrast, to the best of our knowledge, there are few works on power management with Hetnet. In this paper, we take EVs with recharged batteries into account and present the optimal strategy in Hetnet. Firstly, considering the relationship among them composed of EV users, CSOs, and SGOs, we develop different utility functions with the load uncertainty and BER in Hetnet. In order to obtain the optimal strategy, we propose a four-stage Stackelberg game scheme, in which SGO is set as the leader, while both EV users and CSOs are set as followers. Through a back induction method, we get the SE in the proposed game scheme, by proving its existence and uniqueness. Using the presented iterative search algorithm, the optimal solutions are achieved with maximum profits in this charging system. The performance of our proposed algorithm is shown in the simulation results. It demonstrates the effectiveness of our proposal, through which it can benefit each player in the game. 


\section{System Model}

\subsection{Network Model}

In this section, we design the network model in Hetnet to describe the communication in the charging system. Then, based on the trading relationship among EV users, CSOs, and SGOs, we develop the utility function of each. Driven by maximum profits, a four-stage Stackelberg game scheme is proposed to study the optimal strategy.

As shown in Figure 1, we assume that EV users equipped with on-board units (OBU) can communicate with each other, while they can share real-time information by road side units (RSU). In order to serve EV users conveniently, the base station (BS) deployed by CSOs is also used to communicate with EV users in Hetnet, including the power price, power demand, and total power supply. Based on Hetnet, EV users can be charged with less waiting time than that without communication technology. In this charging system, we assume that EV user $k$ will be charged by COS $m$, which is supplied by SGO $i$, denoted as $\forall k \in \mathcal{J}_{m}, \mathcal{J}_{m}=\left\{1, \ldots, J_{m}\right\}$, and $\forall m \in \mathcal{M}, \mathcal{M}=\{1, \ldots, M\}$, respectively. In this paper, $\mathrm{SGO} i$ is assumed to supply power to multiple CSOs.

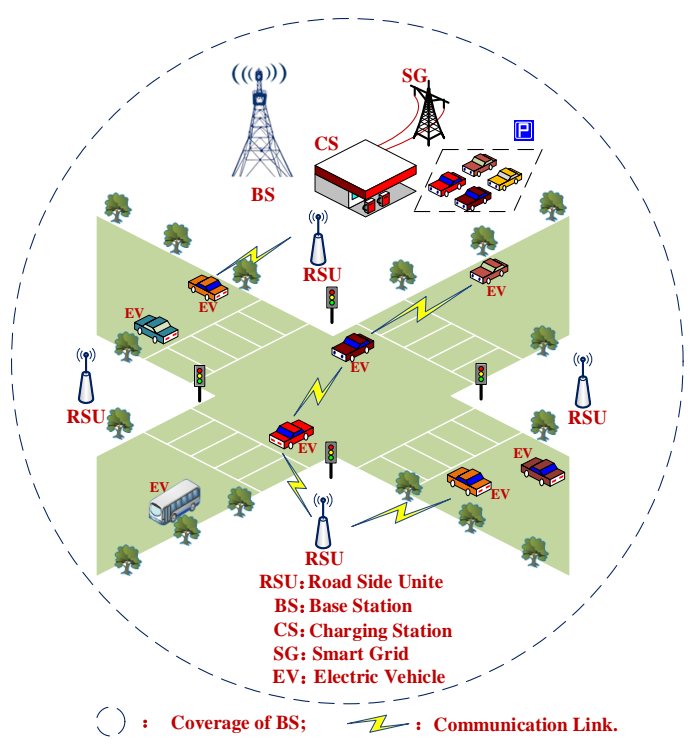

Figure 1. Network model in the heterogeneous network.

\subsection{Utility Model for Electric Vehicles}

In the power market, one of the economic criteria is clients' satisfaction with respect to the supplied power. Here, we suppose that the utility function can be defined by the difference between $\mathrm{EVs}^{\prime}$ satisfaction and the payment for $\operatorname{COS} m$, which is expressed by:

$$
\begin{aligned}
& G_{E V}(\boldsymbol{X})=\sum_{k=1}^{J_{m}}\left(U_{1}\left(x_{k, m}, p_{m}^{i}\right)-C_{1}\left(x_{k, m}, p_{m}^{i}\right)\right) \\
& \text { s.t. } x_{k, m}^{\min } \leq x_{k, m} \leq x_{k, m}^{\max }
\end{aligned}
$$

Here, $p_{m}^{i}$ denotes the power price offered by CSO $m ; \boldsymbol{X}$ is the set of power demands, $\boldsymbol{X}=$ $\left(x_{1, m}, \ldots, x_{J_{m}, m}\right) ; U_{1}\left(x_{k, m}, p_{m}^{i}\right)$ denotes the satisfaction; $C_{1}\left(x_{k, m}, p_{m}^{i}\right)$ denotes the payment for charging service. $x_{k, m}$ denotes the power demand of EV user $k$ with the constraints of a proper upper limit $x_{k, m}^{\max }$ and a proper lower limit $x_{k, m}^{\mathrm{min}}$.

Based on the requirement of charging service, EV users can acquire higher satisfaction with less waiting time and a lower power price. Thus, $U_{1}\left(x_{k, m}, p_{m}^{i}\right)$ should monotonically decrease on waiting 
time $W_{m}$ and $p_{m}^{i}$, respectively. In contrast, it will increase on the amount of charging power $x_{k, m}$. According to [33], we design $U_{1}\left(x_{k, m}, p_{m}^{i}\right)$ as a logarithmic function shown by:

$$
U_{1}\left(x_{k, m}, p_{m}^{i}\right)=\bar{\gamma}_{1} \frac{x_{k, m}}{\zeta_{m}}+\left(A_{k}-\frac{W_{m}}{W_{\max }} p_{m}^{i}\right) \ln \left(1+x_{k, m}\right)
$$

where $\bar{\gamma}_{1}$ denotes the preset economic parameter; $\varsigma_{m}$ is the discount of charging service; $A_{k}$ is a preset parameter to ensure this function is non-negative; $W_{m}$ is the waiting time in CSO $m$, which is bounded by the maximum waiting time $W_{\max }$.

Meanwhile, EV user $k$ will pay for the charging power supplied from CSOs, including the cost in the driving distance. Therefore, $C_{1}\left(x_{k, m}, p_{m}^{i}\right)$ is defined by:

$$
C_{1}\left(x_{k, m}, p_{m}^{i}\right)=p_{m}^{i} x_{k, m}+\delta_{k, m} d_{k, m}
$$

where $\delta_{k, m}$ denotes the average economic parameter for EV user $k ; d_{k, m}$ is EV user $k^{\prime}$ s driving distance to $\mathrm{CSO} m$ in Hetnet.

Then, substituting (2) and (3) into (1), we have:

$$
\begin{aligned}
G_{E V}(\boldsymbol{X}) & =\sum_{k=1}^{J_{m}}\left[\bar{\gamma}_{1} \frac{x_{k, m}}{\zeta_{m}}+\left(A_{k}-\frac{W_{m}}{W_{\max }} p_{m}^{i}\right) \ln \left(1+x_{k, m}\right)\right. \\
& \left.-p_{m}^{i} x_{k, m}-\delta_{k, m} d_{k, m}\right]
\end{aligned}
$$

\subsection{Utility Model for Charging Station Operators}

Meeting the power demand, each CSO can acquire profits through selling the power to EV users. In order to maximize their profits, CSOs will analyze how to decide the optimal strategy. Here, we assume that the unstable communication in Hetnet leads to the loss of power in transmission, which implies that the power demand of EV users received by CSOs will be $\hat{x}_{k, m}=\left(1-\zeta_{m}\right) x_{k, m}+\zeta_{m} \theta_{m} . \zeta_{m}$ is the probability of the loss of power and $\theta_{m}$ denotes the load uncertainty in this charging system. $\theta_{m}$ is supposed to be a zero-mean random variable with variance $\sigma_{\theta}^{2}$. The utility function of CSO $m$ with its constraints is defined by:

$$
\begin{aligned}
& G_{C S}(\boldsymbol{Y})=\sum_{m=1}^{M}\left(\sum_{k=1}^{I_{m}} R_{k, m}\left(p_{m}^{i}, x_{k, m}\right)-C_{2}\left(y_{m, i}, p_{i}\right)\right) \\
& \text { s.t. } \sum_{k=1}^{I_{m}} \hat{x}_{k, m} \leq y_{m, i}
\end{aligned}
$$

Here, the set of CSOs' power demand is $\boldsymbol{Y}$, denoted by $\boldsymbol{Y}=\left(y_{1, i,}, y_{2, i}, \ldots, y_{M, i}\right) . C_{2}\left(y_{m, i}, p_{i}\right)$ denotes the cost for the power supply $y_{m, i}$ from SGO $i$, which can be designed by:

$$
\begin{aligned}
R_{k, m}\left(p_{m}^{i}, x_{k, m}\right) & =\left(p_{m}^{i}-\frac{\bar{\gamma}_{1}}{\varsigma_{m}}\right) \hat{x}_{k, m} \\
C_{2}\left(y_{m, i}, p_{i}\right) & =p_{i} y_{m, i}
\end{aligned}
$$

In order to ensure the balance between the power demand and supply, we define that the probability of charging power supplied to EV users exceeding the power bought will be less than a small requirement level, in which a threshold is used to denote the amount of EV users' power demand beyond the power supply. Thus, according to (7), it can be further expressed by: 


$$
\operatorname{Pr}\left\{\sum_{k=1}^{J_{m}}\left(\eta_{m} x_{k, m}+\zeta_{m} \theta_{m}\right)-y_{m, i} \geq \tau_{m}\right\} \leq \vartheta_{m}
$$

Here, $\eta_{m}$ is the quality of service in Hetnet QoS, denoted by $\eta_{m}=1-\zeta_{m} ; \tau_{m}$ is the threshold value; $\vartheta_{m}$ is a smaller value as the requirement level.

Therefore, substituting (8) and (9) into (6), we have:

$$
G_{C S}(\boldsymbol{Y})=\sum_{m=1}^{M}\left[\left(p_{m}^{i}-\frac{\bar{\gamma}_{1}}{\zeta_{m}}\right) \sum_{k=1}^{J_{m}}\left(\eta_{m} x_{k, m}+\zeta_{m} \theta_{m}\right)-p_{i} y_{m, i}\right]
$$

\subsection{Utility Model for Smart Grid Operators}

Based on the above statement, SGOs can also obtain the payoff through selling power to CSOs, besides the necessary investment in generating electricity. We formulate the utility function as the difference between the payoff and investment, expressed by:

$$
\begin{aligned}
& G_{S G}\left(p_{i}, \boldsymbol{Y}\right)=\sum_{m=1}^{M}\left(R_{m, i}\left(p_{i}, y_{m, i}\right)-C_{3}\left(y_{m, i}\right)\right) \\
& \text { s.t. } \sum_{m=1}^{M} y_{m, i} \leq \Theta
\end{aligned}
$$

where $p_{i}$ is the power price offered by SGO $i ; R_{m, i}\left(p_{i}, y_{m, i}\right)$ is SGO $i$ 's payoff; $C_{3}\left(y_{m, i}\right)$ denotes the investment for power supply $y_{m, i}$. Based on $(8)$ and $[24,25]$, we have:

$$
\begin{aligned}
R_{m, i}\left(p_{i}, y_{m, i}\right) & =p_{i} y_{m, i} \\
C_{3}\left(y_{m, i}\right) & =\frac{a_{i}}{2} y_{m, i}^{2}+b_{i} y_{m, i}+\omega_{i}
\end{aligned}
$$

where $a_{i}$ denotes the variable acceleration of the cost function curve; $b_{i}$ is the rate of the variety of the cost function curve; $\omega_{i}$ denotes the fixed cost.

Therefore, substituting (14) and (15) into (12), it can be rewritten as:

$$
G_{S G}\left(p_{i}, \boldsymbol{Y}\right)=\sum_{m=1}^{M}\left(p_{i} y_{m, i}-\frac{a_{i}}{2} y_{m, i}^{2}-b_{i} y_{m, i}-\omega_{i}\right)
$$

\subsection{A Four-Stage Stackelberg Game Model}

We study and analyze the trading interaction among EV users, CSOs, and SGOs, while the utility function of each in this charging system is developed, respectively. Due to selfishness, each of them in this system aims to maximize its own profits without consideration of others' payoff. However, with rationality, each of them studies how to maximize its own profits, based on others' decisions in this system. Therefore, in order to decide the optimal strategy, we adopt game theory to study the charging problem, where a four-stage Stackelberg game scheme is developed in Figure 2. In Stage I, SGO $i$ as the leader will determine the power price $p_{i}$ and broadcast this information to CSOs. The proper amount of power $y_{m, i}(\forall m \in \mathcal{M})$ will be decided by each CSO in Stage II, given $p_{i}$ in Stage I. Then, in order to obtain higher revenue, CSO $m$ will decide its price $p_{m, i}$ and the supply power to EV users in Stage III. At last, in Stage IV, EV user $k\left(\forall k \in \mathcal{J}_{m}\right)$ will decide its power demand $x_{k, m}$ based on $p_{m, i}$. 


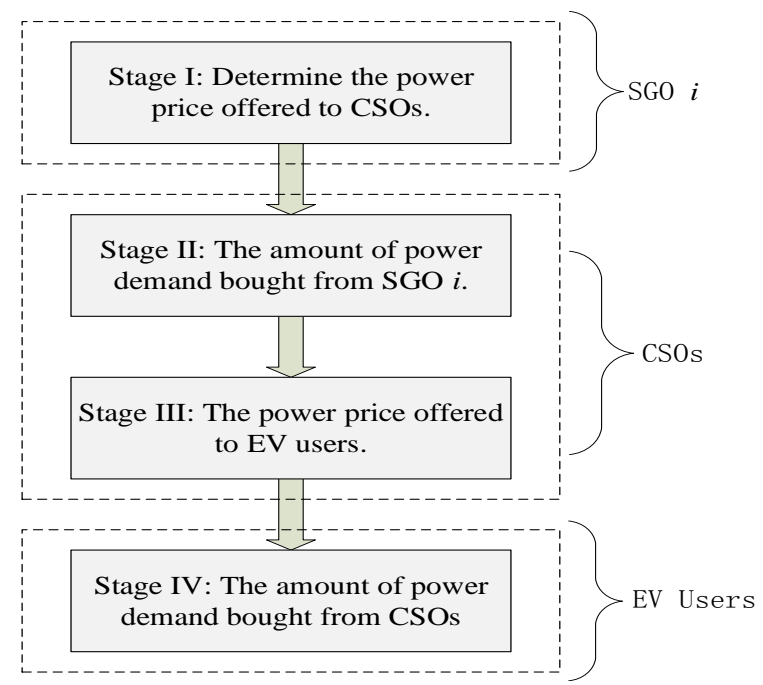

Figure 2. Four-stage Stackelberg game scheme. CSO, charging station operator; SGO, smart grid operator.

For EV users, the relationship among them is non-cooperative and competitive. The objection of each EV user is to maximize its profits based on the price offered by CSOs. Thus, this problem can be formulated as an optimization problem shown by:

$$
\boldsymbol{X}_{m}^{*}=\arg \max G_{E V}\left(\boldsymbol{X}_{m}\right)
$$

Here, the set of optimal power demand supplied by CSO $m$ is $\boldsymbol{X}_{m}^{*}$, denoted by $\boldsymbol{X}_{m}^{*}=$ $\left(x_{1, m}^{*}, x_{2, m}^{*}, \ldots, x_{J_{m}, m}^{*}\right)$.

Then, CSOs aim to make the optimal pricing plans, with consideration of EV users' power demand bounded by the constraints shown in (2). Similarly, this optimization problem is formulated as:

$$
p_{m}^{i *}=\arg \max G_{C S}\left(\boldsymbol{Y}, p_{m}^{i}\right)
$$

where $p_{m}^{i *}$ is the optimal pricing strategy offered by CSO $m$.

In addition, each CSO will determine the amount of power supplied from SGOs with maximum profits. Following the optimal pricing strategy $p_{m}^{i *}$, CSO $m$ studies how to obtain the power demand from SGO $i$, given the power price $p_{i}$. In this case, the optimization problem is formulated as:

$$
y_{m, i}^{*}=\arg \max G_{C S}\left(\boldsymbol{Y}, p_{m}^{i}\right)
$$

where $y_{m, i}^{*}$ is the optimal power demand strategy for CSO $m$ powered by SGO $i$.

Finally, driven by maximum profits, SGOs analyze how to make decisions on the optimal pricing strategy. Following (19), this problem is formulated as an optimization problem shown by:

$$
p_{i}^{*}=\arg \max G_{S G}\left(p_{i}, \boldsymbol{Y}\right)
$$

where $p_{i}^{*}$ is the optimal pricing plan of SGO $i$ offered to CSOs.

Through the above analysis, the optimal solution can be decided by the proposed four-stage game-theoretical scheme. In this game, SE will be solved to maximize their own profits. In this case, each of them will not tend to change its optimal decisions.

\section{Four-Stage Stackelberg Game Analysis}

In this section, we will analyze the game-theoretical scheme and obtain the SE through backward induction. First of all, we discuss how EV users adjust their power demand to maximize their profits 
in Stage IV. Then, based on EV users' power demand, we will analyze how CSOs make decisions on the power pricing for EV users and the amount of power demand from SGO in Stages III and II, respectively. At last, we exploit how SGO decides the powering strategy in Stage I.

\subsection{Power Demand of EV Users in Stage IV}

Based on the price broadcast by CSOs, EV users make optimal decisions to maximize their profits, besides the necessary payment for power demand. In order to determine the optimal strategy, this can be achieved through solving the following optimization problem shown by:

$$
\begin{aligned}
& \max _{\forall k \in J_{m}, x_{k, m} \geq 0} G_{E V}(\boldsymbol{X}) \\
= & \sum_{k=1}^{J_{m}}\left[\bar{\gamma}_{1} \frac{x_{k, m}}{\varsigma_{m}}+\left(A_{k}-\frac{W_{m}}{W_{\max }} p_{m}\right) \ln \left(1+x_{k, m}\right)\right. \\
& \left.-p_{m} x_{k, m}-\delta_{k, m} d_{k, m}\right]
\end{aligned}
$$

Here, without loss of generality, $p_{m}$ is usually decided to be larger than $\frac{\bar{\gamma}_{1}}{\zeta_{m}}$, e.g., $p_{m}>\frac{\bar{\gamma}_{1}}{\zeta_{m}}$. Thus, we take the first derivation of $G_{E V}(\boldsymbol{X})$ with respect to $x_{k, m}$ as follows.

$$
\frac{\partial G_{E V}(\boldsymbol{X})}{\partial x_{k, m}}=\sum_{k=1}^{J_{m}}\left(\frac{A_{k}-\frac{W_{m}}{W_{\max }} p_{m}}{1+x_{k, m}}+\frac{\bar{\gamma}_{1}}{\zeta_{m}}-p_{m}\right)
$$

Then, taking the second derivation of $G_{E V}(X)$ with respect to $x_{k, m}$, we have:

$$
\frac{\partial^{2} G_{E V}(\boldsymbol{X})}{\partial x_{k, m}^{2}}=-\sum_{k=1}^{J_{m}} \frac{A_{k}-\frac{W_{m}}{W_{\max }} p_{m}}{\left(1+x_{k, m}\right)^{2}}
$$

Since $A_{k}-\frac{W_{m}}{W_{\max }} p_{m}$ is larger than zero in (3), we can know that $\frac{\partial^{2} G_{E V}(\boldsymbol{X})}{\partial x_{k, m}^{2}}<0$, which implies that the first derivation of $G_{E V}(X)$ is strictly decreasing on $x_{k, m}$. Thus, we divide it into two cases to analyze the optimal strategy.

Case 1: CSOs will announce the lower price to EV users to be charged.

If the power price $p_{m}$ is lower than $\frac{\left(A_{k} \zeta_{m}+\bar{\gamma}_{1}\right) W_{\max }}{\left(W_{m}+W_{\max }\right) \zeta_{m}}$ in (22), we have:

$$
\begin{aligned}
& \lim _{x_{k, m} \rightarrow 0} \frac{\partial G_{E V}(\boldsymbol{X})}{\partial x_{k, m}}=\sum_{k=1}^{J_{m}}\left[A_{k}+\frac{\bar{\gamma}_{1}}{\varsigma_{m}}-p_{m}\left(\frac{W_{m}}{W_{\max }}+1\right)\right]>0 \\
& \lim _{x_{k, m} \rightarrow \infty} \frac{\partial G_{E V}(\boldsymbol{X})}{\partial x_{k, m}}=-\sum_{k=1}^{J_{m}}\left(p_{m}-\frac{\bar{\gamma}_{1}}{\zeta_{m}}\right)<0
\end{aligned}
$$

Therefore, based on the results in (24) and (25), this implies that the utility function is firstly increasing with the increase of $x_{k, m}$. Then, it continuously decreases with the increase of $x_{k, m}$. It will prove that the utility function in (21) is a concave function and that the optimal strategy exists. Correspondingly, we can obtain the optimal strategy.

Case 2: CSOs will announce the larger price to EV users to be charged. 
If the power price $p_{m}$ is not less than $\frac{\left(A_{k} \zeta_{m}+\bar{\gamma}_{1}\right) W_{\max }}{\left(W_{m}+W_{\max }\right) \zeta_{m}}$, similar to the method used in Case 1, we have:

$$
\begin{aligned}
& \lim _{x_{k, m} \rightarrow 0} \frac{\partial G_{E V}(\boldsymbol{X})}{\partial x_{k, m}}=\sum_{k=1}^{J_{m}}\left[A_{k}-p_{m}\left(\frac{W_{m}}{W_{\max }}+1\right)-\frac{\bar{\gamma}_{1}}{\zeta_{m}}\right] \leq 0 \\
& \lim _{x_{k, m} \rightarrow \infty} \frac{\partial G_{E V}(\boldsymbol{X})}{\partial x_{k, m}}=-\sum_{k=1}^{J_{m}}\left(p_{m}-\frac{\bar{\gamma}_{1}}{\zeta_{m}}\right)<0
\end{aligned}
$$

From (26) and (27), we can know that the utility function in (21) decreases with the increase of $x_{k, m}$, which implies that the optimal strategy also exists. The optimal strategy for EV users is:

$$
x_{k, m}^{*}=0
$$

Based on the above analysis, we can know that $G_{E V}(\boldsymbol{X})$ is a strictly concave function on $\boldsymbol{X}$. The optimal strategy will be obtained through solving $\frac{\partial G_{E V}(\boldsymbol{X})}{\partial x_{k, m}}=0$, and we have:

$$
x_{k, m}^{*}=\left\{\begin{array}{cc}
\frac{\left(A_{k} W_{\max }-p_{m} W_{m}\right) \varsigma_{m}}{\left(\zeta_{m} p_{m}-\bar{\gamma}_{1}\right) W_{\max }}-1, & \text { if } p_{m}<\frac{\left(A_{k} \zeta_{m}+\bar{\gamma}_{1}\right) W_{\max }}{\left(W_{m}+W_{\max }\right) \zeta_{m}} \\
0, & \text { others }
\end{array}\right.
$$

Due to the requirement of protecting batteries' healthy, each EV user's power demand is larger than zero, which means that the power demand of EV users is larger than zero. As a result, the optimal strategy is obtained as follows.

$$
x_{k, m}^{*}=\frac{\left(A_{k} W_{\max }-p_{m} W_{m}\right) \varsigma_{m}}{\left(\varsigma_{m} p_{m}-\bar{\gamma}_{1}\right) W_{\max }}-1
$$

\subsection{Power Price Offered by Charging Station Operators in Stage III}

In this subsection, we will study how CSOs make pricing decisions to improve their profits, on the condition that the power demand of EV users can be decided in Stage IV. Considering the fixed expense and the power price offered by SGO, CSOs should adjust their power price for EV users driven by more profits. Due to the impact of uncertain load, we have:

$$
\begin{aligned}
& \mathbb{E}\left\{G_{C S}\left(\boldsymbol{Y}, \boldsymbol{P}^{i}\right)\right\} \\
& =\sum_{m=1}^{M}\left\{\sum_{k=1}^{J_{m}} \eta_{m}\left[\frac{A_{k} W_{\max }-p_{m}\left(W_{m}+W_{\max }\right)}{W_{\max }}+\frac{\bar{\gamma}_{1}}{\zeta_{m}}\right]-p_{i} y_{m, i}-\epsilon_{m} L_{m}\right\} \\
& \text { s.t. } \operatorname{Pr}\left\{\sum_{k=1}^{J_{m}}\left[\eta_{m} x_{k, m}^{*}+\zeta_{m} \theta_{m}\right]-y_{m, i} \geq \tau_{m}\right\} \leq \vartheta_{m}
\end{aligned}
$$


Here, it is worthy to note that (31) implies that the utility function of CSOs is monotonically decreasing with the increase of $p_{m}$, which means that the optimal strategy will exist on the condition that the constraints can be known. Substituting (30) into (32), we have:

$$
\begin{aligned}
& \operatorname{Pr}\left\{\sum_{k=1}^{J_{m}}\left(\eta_{m} x_{k, m}^{*}+\zeta_{m} \theta_{k, m}\right)-y_{m, i} \geq \tau_{m}\right\} \leq \vartheta_{m} \\
& \Longrightarrow \operatorname{Pr}\left\{\sum_{k=1}^{J_{m}} \theta_{k, m} \geq \frac{1}{\zeta_{m}}\left(y_{m, i}+\tau_{m}-\sum_{k=1}^{I_{m}} \eta_{m} x_{k, m}^{*}\right)\right\} \leq \vartheta_{m} \\
& \Longrightarrow \frac{1}{2} \operatorname{Pr}\left\{\zeta_{m}\left|\sum_{k=1}^{J_{m}} \theta_{k, m}\right| \geq y_{m, i}+\tau_{m}-\sum_{k=1}^{I_{m}} \eta_{m} x_{k, m}^{*}\right\} \leq \vartheta_{m} \\
& \Longrightarrow \frac{1}{2} \frac{\left(\zeta_{m} \sigma_{\theta}\right)^{2} J_{m}}{\left(y_{m, i}+\tau_{m}-\sum_{k=1}^{J_{m}} \eta_{m} x_{k, m}^{*}\right)^{2}} \leq \vartheta_{m} \\
& \Longrightarrow p_{m} \geq \frac{\eta_{m} \sum_{k=1}^{J_{m}}\left(A_{k}-\frac{\bar{\gamma}_{1} W_{m}}{\zeta_{m} W_{\max }}\right)}{y_{m, i}+\tau_{m}-\sqrt{\frac{I_{m}}{2 \vartheta_{m}}} \zeta_{m} \sigma_{\theta}+\eta_{m} J_{m}\left(\frac{W_{m}+W_{\max }}{W_{\max }}\right)}+\frac{\bar{\gamma}_{1}}{\zeta_{m}}
\end{aligned}
$$

which implies that the optimal price is shown by:

$$
p_{m}^{*}=\frac{\bar{\gamma}_{1}}{\zeta_{m}}+\frac{\eta_{m} \sum_{k=1}^{I_{m}}\left(A_{k}-\frac{\bar{\gamma}_{1} W_{m}}{\zeta_{m} W_{\max }}\right)}{y_{m, i}+\tau_{m}-\sqrt{\frac{I_{m}}{2 \vartheta_{m}}} \zeta_{m} \sigma_{\theta}+\eta_{m} J_{m}\left(\frac{W_{m}+W_{\max }}{W_{\max }}\right)}
$$

\subsection{Power Supply of Charging Station Operators in Stage II}

Based on the price and power demand for EV users, the objection of operators will improve their profits besides the payment for electricity retailers. In this stage, CSOs decide the power supply to be procured from SGO. Substituting (34) into (11), we have:

$$
\begin{aligned}
\mathbb{E}\left\{G_{C S}\left(\boldsymbol{Y}, \boldsymbol{P}^{i}\right)\right\} \\
=\sum_{m=1}^{M}\left\{\sum_{k=1}^{J_{m}}\left[\eta_{m} A_{k}-\frac{\eta_{m}^{2} \sum_{k=1}^{J_{m}}\left(A_{k}-\frac{\bar{\gamma}_{1} W_{m}}{\varsigma_{m} W_{\max }}\right)}{\frac{y_{m, i}+\Omega_{m}}{W_{m}+W_{\max }} W_{\max }}\right]\right. \\
\left.\quad-\frac{\bar{\gamma}_{1} \eta_{m} J_{m}\left(W_{m}+W_{\max }\right)}{\varsigma_{m} W_{\max }}-p_{i} y_{m, i}\right\}
\end{aligned}
$$

where:

$$
\Omega_{m}=\tau_{m}-\sqrt{\frac{J_{m}}{2 \vartheta_{m}}} \zeta_{m} \sigma_{\theta}+\eta_{m} J_{m}\left(\frac{W_{m}+W_{\max }}{W_{\max }}\right)
$$


In order to obtain the optimal solution in (35), we should exploit and discuss its existence given the price offered by SGO. Similar to the method in Stage IV, we take the first derivative of $\mathbb{E}\left\{G_{C S}\left(\boldsymbol{Y}, \boldsymbol{P}^{i}\right)\right\}$ with respect to $y_{m, i}$ as follows.

$$
\frac{\partial \mathbb{E}\left\{G_{C S}\left(\boldsymbol{Y}, \boldsymbol{P}^{i}\right)\right\}}{\partial y_{m, i}}=\sum_{m=1}^{M}\left[\frac{\eta_{m}^{2} J_{m}\left(W_{m}+W_{\max }\right) \sum_{k=1}^{I_{m}}\left(A_{k}-\frac{\bar{\gamma}_{1} W_{m}}{\varsigma_{m} W_{\max }}\right)}{W_{\max }\left(y_{m, i}+\Omega_{m}\right)^{2}}-p_{i}\right]
$$

Following, taking the second derivative of $\mathbb{E}\left\{G_{C S}\left(Y, P^{i}\right)\right\}$ with respect to $y_{m, i}$, we have:

$$
\frac{\partial^{2} \mathbb{E}\left\{G_{C S}\left(\boldsymbol{Y}, \boldsymbol{P}^{i}\right)\right\}}{\partial y_{m, i}^{2}}=-\sum_{m=1}^{M}\left[\frac{2 \eta_{m}^{2} J_{m}\left(W_{m}+W_{\max }\right) \sum_{k=1}^{J_{m}}\left(A_{k}-\frac{\bar{\gamma}_{1} W_{m}}{\zeta_{m} W_{\max }}\right)}{W_{\max }\left(y_{m, i}+\Omega_{m}\right)^{3}}\right]
$$

Since $A_{k}>\frac{W_{m}}{W_{\max }} p_{m}$ and $p_{m}>\frac{\bar{\gamma}_{1}}{\zeta_{m}}$, we can know that $A_{k}>\frac{\bar{\gamma}_{1} W_{m}}{\zeta_{m} W_{\max }}$. In addition, due to the non-negative parameters in (35), it means that the second derivative of $\mathbb{E}\left\{G_{C S}\left(\boldsymbol{Y}, \boldsymbol{P}^{i}\right)\right\}$ is less than zero. Based on (35)-(38), this implies that the optimal solution can be achieved when the following conditions is satisfied.

$$
\begin{aligned}
& \lim _{y_{m, i} \rightarrow 0} \frac{\partial \mathbb{E}\left\{G_{C S}\left(\boldsymbol{Y}, \boldsymbol{P}^{i}\right)\right\}}{\partial y_{m, i}}>0 \\
& \Longrightarrow \sum_{m=1}^{M}\left[\frac{\eta_{m}^{2} J_{m}\left(W_{m}+W_{\max }\right) \sum_{k=1}^{I_{m}}\left(A_{k}-\frac{\bar{\gamma}_{1} W_{m}}{\varsigma_{m} W_{\max }}\right)}{W_{\max } \Omega_{m}^{2}}\right]-M p_{i}>0 \\
& \Longrightarrow p_{i}<\frac{1}{M} \sum_{m=1}^{M}\left[\frac{\eta_{m}^{2} J_{m}\left(W_{m}+W_{\max }\right)}{W_{\max } \Omega_{m}^{2}}\left(\sum_{k=1}^{J_{m}} A_{k}-\frac{\bar{\gamma}_{1} J_{m} W_{m}}{\zeta_{m} W_{\max }}\right)\right]
\end{aligned}
$$

and:

$$
\lim _{y_{m, i} \rightarrow \infty} \frac{\partial \mathbb{E}\left\{G_{C S}\left(x_{k, m}, p_{k, m}\right)\right\}}{\partial y_{m, i}}=-M p_{i}<0
$$

Thus, the utility function of $\mathbb{E}\left\{G_{C S}\left(\boldsymbol{Y}, \boldsymbol{P}^{i}\right)\right\}$ is a concave function when both the inequalities (39) and (40) are satisfied. It proves that the optimal strategy exists, which can be achieved as follows.

$$
\begin{aligned}
& \frac{\partial \mathbb{E}\left\{G_{C S}\left(\boldsymbol{Y}, \boldsymbol{P}^{i}\right)\right\}}{\partial y_{m, i}}=0 \\
& \Longleftrightarrow \frac{\eta_{m}^{2} J_{m}\left(W_{m}+W_{\max }\right) \sum_{k=1}^{J_{m}}\left(A_{k}-\frac{\bar{\gamma}_{1} W_{m}}{\zeta_{m} W_{\max }}\right)}{W_{\max }\left(y_{m, i}+\Omega_{m}\right)^{2}}=p_{i} \\
& \Longleftrightarrow y_{m, i}^{*}=\sqrt{\frac{\eta_{m}^{2} J_{m}\left(W_{m}+W_{\max }\right) \sum_{k=1}^{I_{m}}\left(A_{k}-\frac{\bar{\gamma}_{1} W_{m}}{\zeta_{m} W_{\max }}\right)}{W_{\max } p_{i}}}-\Omega_{m}
\end{aligned}
$$


Meanwhile, based on (34) and (41), we can obtain the following results.

$$
\begin{aligned}
& p_{m}^{*}<\frac{\left(A_{k} \zeta_{m}+\bar{\gamma}_{1}\right) W_{\max }}{\left(W_{m}+W_{\max }\right) \zeta_{m}} \\
\Longrightarrow & \frac{\bar{\gamma}_{1}}{\zeta_{m}}+\frac{\eta_{m} \sum_{k=1}^{J_{m}}\left(A_{k}-\frac{\bar{\gamma}_{1} W_{m}}{\zeta_{m} W_{\max }}\right)}{y_{m, i}+\Omega_{m}}<\frac{\left(A_{k} \zeta_{m}+\bar{\gamma}_{1}\right) W_{\max }}{\left(W_{m}+W_{\max }\right) \zeta_{m}} \\
\Longrightarrow & \frac{\eta_{m} \zeta_{m}\left(W_{m}+W_{\max }\right) \sum_{k=1}^{J_{m}}\left(A_{k}-\frac{\bar{\gamma}_{1} W_{m}}{\zeta_{m} W_{\max }}\right)}{A_{k} W_{\max } \zeta_{m}-\bar{\gamma}_{1} W_{m}}-\Omega_{m}<y_{m, i}^{*} \\
\Longrightarrow & p_{i}<\frac{J_{m}\left(\zeta_{m} W_{\max } \min A_{k}-\bar{\gamma}_{1} W_{m}\right)^{2}}{\zeta_{m}^{2} W_{\max }\left(W_{m}+W_{\max }\right) \sum_{k=1}^{J_{m}}\left(A_{k}-\frac{\bar{\gamma}_{1} W_{m}}{\zeta_{m} W_{\max }}\right)}
\end{aligned}
$$

In addition, driven by more profits, the power price for EV users to be charged is larger than that offered by SGO when the same amount of power procured from CSO will be sold to EV users by CSOs, e.g., $p_{m} \geq p_{i}$.

\subsection{Power Price Offered by the Smart Grid Operator in Stage I}

Given the power price and power demand offered by CSOs, SGO should decide the power price for CSOs to obtain more profits. This pricing problem can be formulated as an optimization problem. Based on (16), (41) and (42), this optimization problem can be expressed by:

$$
\begin{aligned}
& \max G_{S G}(\boldsymbol{P})=\sum_{m=1}^{M}\left(p_{i} y_{m, i}^{*}-\frac{a_{i}}{2} y_{m, i}^{* 2}-b_{i} y_{m, i}^{*}-\boldsymbol{\omega}_{i}\right) \\
& \text { s.t. } \sum_{m=1}^{M} y_{m, i}^{*} \leq \Theta
\end{aligned}
$$

Substituting (41) into (43), we take the second derivative of $G_{S G}(P)$ with respect to $p_{i}$, and we have:

$$
\begin{aligned}
& \frac{\partial^{2} G_{S G}(\boldsymbol{P})}{\partial p_{i}^{2}} \\
& =-\sum_{m=1}^{M} \sqrt{\frac{\eta_{m}^{2} J_{m}\left(W_{m}+W_{\max }\right) \sum_{k=1}^{J_{m}}\left(A_{k}-\frac{\bar{\gamma}_{1} W_{m}}{\varsigma_{m} W_{\max }}\right)}{W_{\max }}} \\
& \quad \times\left[\frac { a _ { i } } { 4 } \left(4 p_{i}^{-3} \sqrt{\frac{\eta_{m}^{2} J_{m}\left(W_{m}+W_{\max }\right) \sum_{k=1}^{J_{m}}\left(A_{k}-\frac{\bar{\gamma}_{1} W_{m}}{\varsigma_{m} W_{\max }}\right)}{W_{\max }}}\right.\right. \\
& \left.\left.-3 p_{i}^{-\frac{5}{2}} \Omega_{m}\right)+\frac{1}{4} p_{i}^{-\frac{3}{2}}+\frac{3 b_{i}}{4} p_{i}^{-\frac{5}{2}}\right]
\end{aligned}
$$


Then, based on the requirement of CSOs' power supply obtained in Stage II, we have:

$$
\begin{aligned}
& y_{m, i}^{*}=\sqrt{\frac{\eta_{m}^{2} J_{m}\left(W_{m}+W_{\max }\right) \sum_{k=1}^{J_{m}}\left(A_{k}-\frac{\bar{\gamma}_{1} W_{m}}{\zeta_{m} W_{\max }}\right)}{W_{\max } p_{i}}}-\Omega_{m} \geq 0 \\
& \Longrightarrow p_{i} \leq \frac{\eta_{m}^{2} J_{m}\left(W_{m}+W_{\max }\right) \sum_{k=1}^{I_{m}}\left(A_{k}-\frac{\bar{\gamma}_{1} W_{m}}{\varsigma_{m} W_{\max }}\right)}{W_{\max } \Omega_{m}^{2}} \\
& \Longrightarrow \frac{\partial^{2} G_{R e t}\left(p_{i}\right)}{\partial p_{i}^{2}}<0
\end{aligned}
$$

As a result, this implies that $G_{S G}(\boldsymbol{P})$ is a concave function on $p_{i}$, and it also proves that the optimal strategy exists. Similar to the method above, the optimal strategy can be obtained as follows.

$$
\begin{aligned}
& \frac{\partial G_{S G}(\boldsymbol{P})}{\partial p_{i}}=0 \\
& \Longleftrightarrow \frac{1}{2} \sum_{m=1}^{M} p_{i}^{-\frac{1}{2}} \sqrt{\frac{\eta_{m}^{2} J_{m}\left(W_{m}+W_{\max }\right) \sum_{k=1}^{J_{m}}\left(A_{k}-\frac{\bar{\gamma}_{1} W_{m}}{\zeta_{m} W_{\max }}\right)}{W_{\max }}} \\
& \quad \times\left(1+b_{i} p_{i}^{-1}\right)+\frac{p_{i}^{-2} a_{i} \eta_{m}^{2} J_{m}\left(W_{m}+W_{\max }\right)}{2 W_{\max }} \sum_{k=1}^{I_{m}}\left(A_{k}-\frac{\bar{\gamma}_{1} W_{m}}{\zeta_{m} W_{\max }}\right) \\
& =\sum_{m=1}^{M} \frac{a_{i}}{2} p_{i}^{-\frac{3}{2}} \sqrt{\frac{\eta_{m}^{2} J_{m}\left(W_{m}+W_{\max }\right) \sum_{k=1}^{I_{m}}\left(A_{k}-\frac{\bar{\gamma}_{1} W_{m}}{\zeta_{m} W_{\max }}\right)}{W_{\max } \Omega_{m}^{2}}}+\Omega_{m}
\end{aligned}
$$

Then, by solving (47), the SGO can make the optimal pricing strategy with maximum profits.

In conclusion, based on the proposed game-theoretical scheme, we obtain the optimal solutions in the charging system. Due to the analysis mentioned above in the four-stage game, it holds that $G_{E V}\left(\tilde{x}_{k, m}^{*}\right)<G_{E V}\left(x_{k, m}^{*}\right)$ for random $\tilde{x}_{k, m}^{*}$ in Stage IV, satisfying $\forall k \in J_{m}$ and $\forall m \in M$; for random $\tilde{p}_{m}^{*}$, it holds that $G_{C S}\left(\tilde{p}_{m}^{*}\right)<G_{C S}\left(p_{m}^{*}\right)$ in Stage III, satisfying $\forall m \in M$; for random $\tilde{y}_{m, i}^{*}$, it holds that $G_{C S}\left(\tilde{y}_{m, i}^{*}\right)<G_{C S}\left(y_{m, i}^{*}\right)$ in Stage II, satisfying $\forall m \in M$; for random $\tilde{p}_{i}^{*}$, it holds that $G_{S G}\left(\tilde{p}_{i}^{*}\right)<G_{S G}\left(p_{i}^{*}\right)$ in Stage I. Here, $\tilde{x}_{k, m}^{*}, \tilde{p}_{m}^{*}, \tilde{y}_{m, i}^{*}$, and $\tilde{p}_{i}^{*}$ denote other different values, except $x_{k, m}^{*}, p_{m}^{*}, y_{m, i}^{*}$, and $p_{i}^{*}$, respectively. Therefore, it proves that SE exists in our proposed game-theoretical scheme with the optimal solutions shown by (30), (34), (41), and (47), respectively.

\subsection{Algorithm Design to Obtain Stackelberg Equilibrium}

According to the above analysis, it is proven that there is a unique SE in our proposed Stackelberg game, which can be able to maximize the profits in the charging system composed of EV users, CSOs, and SGO. We adopt the backward method to obtain the optimal solutions. In order to simplify the calculation, we propose an iterative algorithm to achieve the SE, which is shown in Algorithm 1. In this presented iterative algorithm, we induce the convergence criteria $\frac{\left\|p_{i}^{t}-p_{i}^{t-1}\right\|}{\left\|p_{i}^{t-1}\right\|} \leq \varrho$ to obtain the precise solutions, in which $\varrho$ is a small threshold value, i.e., $\varrho=10^{-4}$. 


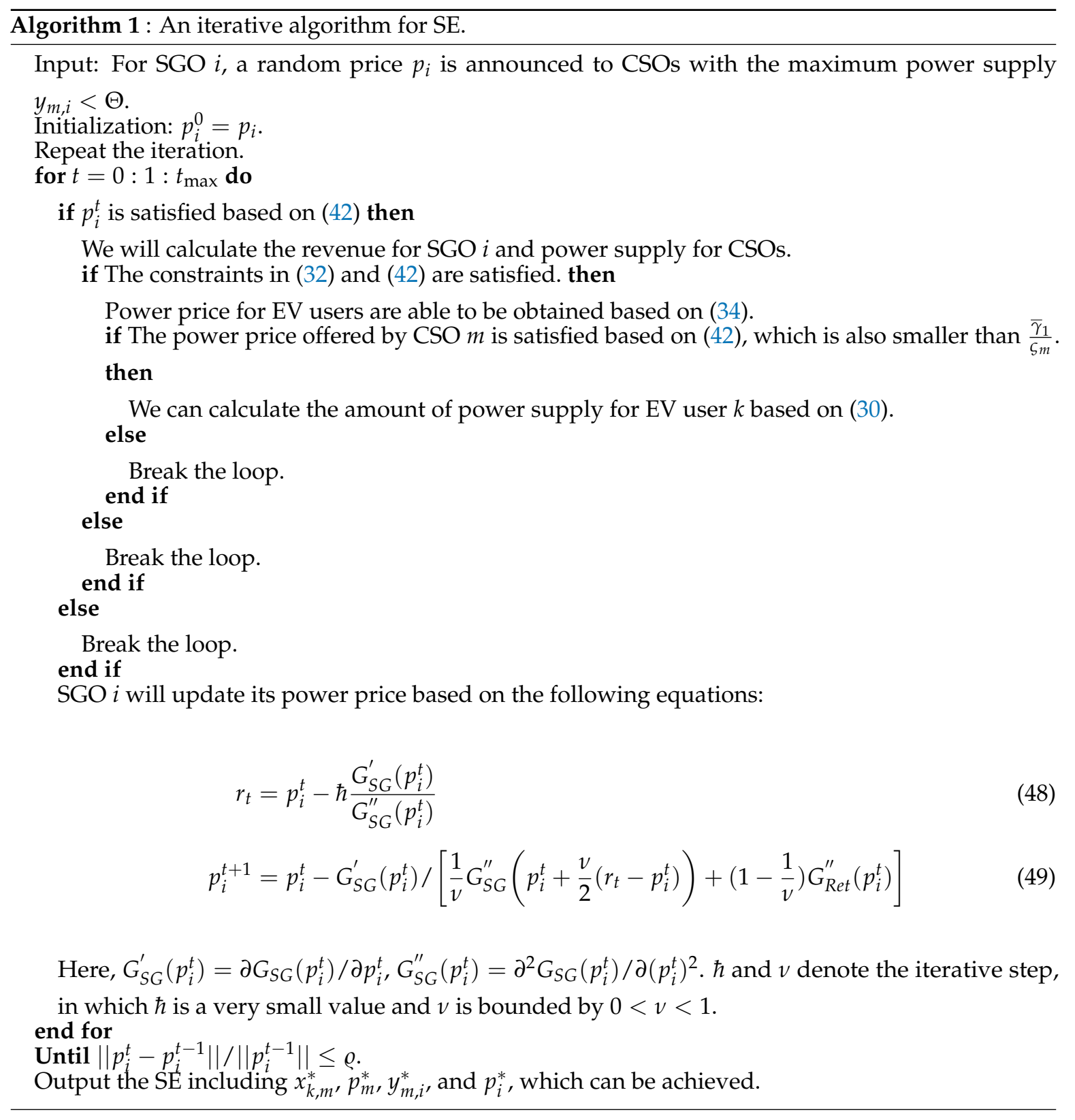

\section{Simulation}

\subsection{Simulation Scenario}

In this section, we demonstrate numerical results to verify the performance of the strategy proposed in this paper. Based on [34], we suppose that BER will be no more than 0.1. The cost for waiting to be charged is $0.3 \$ / \mathrm{h}$ and $0.4 \$ / \mathrm{h}$ in CSO 1 and CSO 2, respectively. Here, assuming that for EV users to be charged arriving at the charging station, $\lambda=4.8 / \mathrm{h}, \mu=1.2 / \mathrm{h}$. Other parameters are set in Table 1 . The total number of EV users to be charged by CSO 1 is 15 , in which $A_{k}(k=1,2,3)$ is equal to 40 . For the rest of the EV users, $A_{k}$ is equal to 50. The average driving distance for each EV user and CSOs is $10 \mathrm{~km}$ and $15 \mathrm{~km}$, respectively. Similarly, the total number charged by CSO 2 is 10 , in which $A_{k}\{k=1,2,3\}$ is equal to 45 . For the rest of the EV users, $A_{k}$ is equal to 50 . Other parameters are set as follows: $\tau_{1}=0.02 \mathrm{MW} \cdot \mathrm{h}, \tau_{2}=0.01 \mathrm{MW} \cdot \mathrm{h}, \sigma_{1}=\sigma_{2}=2, \vartheta_{1}=\vartheta_{2}=0.1$. 
Table 1. Simulation parameters.

\begin{tabular}{cc}
\hline Parameter & Value \\
\hline$W_{\max }$ for EV users to be charged & $\{0.7 \mathrm{~h}, 0.7 \mathrm{~h}\}$ \\
The minimum power demand for EV users & $0 \mathrm{MW} \cdot \mathrm{h}$ \\
The maximum power demand for EV users & $0.5 \mathrm{MW} \cdot \mathrm{h}$ \\
$\bar{\gamma}_{1}$ for each CSO & $2 \$ / \mathrm{h}$ \\
$\bar{\gamma}_{2}$ for each CSO & $0.3 \$ / \mathrm{h}$ \\
Discount charging amount for each EV user & $\{10 \mathrm{MW} \cdot \mathrm{h}\}$ \\
$a_{i}$ for SGO $i$ & 0.5 \\
$b_{i}$ for SGO $i$ & 0.1 \\
$c_{i}$ for SGO $i$ & 80 \\
\hline
\end{tabular}

\subsection{Simulation Results}

Given the preset parameters above, through the proposed game-theoretical scheme strategy, we study how BER plays an important role in the decisions on the optimal strategy. Figure 3 shows the relationship between the optimal price decided by electricity retailer $i$ and BER in Hetnet. Obviously, from the simulation in Figure 3, this demonstrates that the price becomes smaller with the increase of BER, which implies that BER affects the pricing decision.

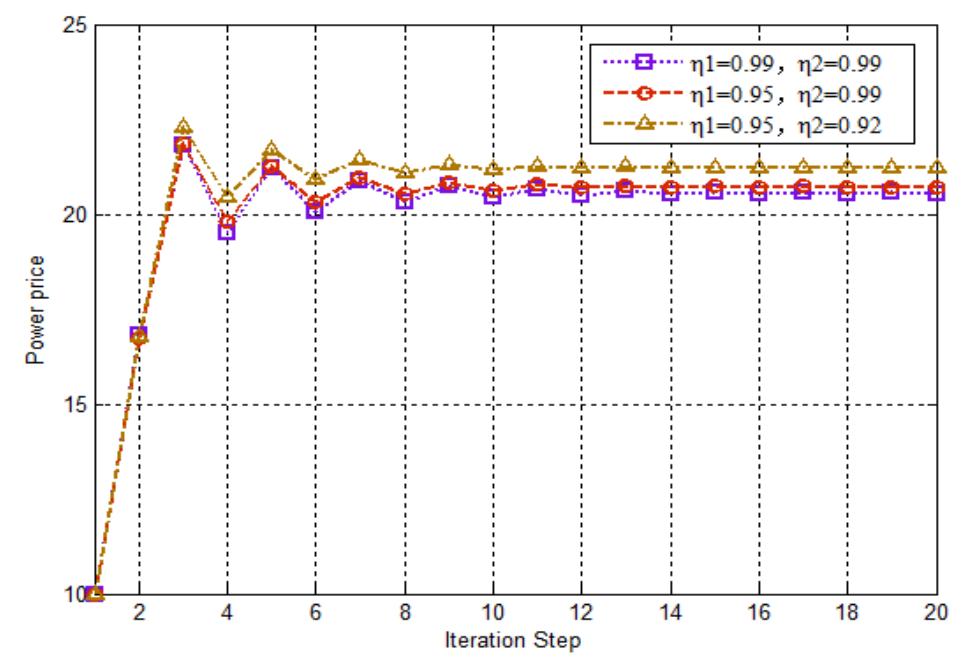

Figure 3. Power price offered by SGO $i$ versus the iteration step, in which its initial price $p_{i}=10$, $C_{1}=C_{2}=3$, and $S_{1}=6, S_{2}=7$, respectively.

For the same example with $\eta_{1}=0.95$ and $\eta_{2}=0.99$ set in Figure 3, Figure 4 illustrates the pricing strategy of electricity retailer $i$ with respect to the iteration step. We can observe that the value of price will converge to a stable value through several iteration steps. In Figure 4, we can also know that the optimal price will be obtained in various cases with different waiting capacities. Similar to Figure 4, Figure 5 illustrates that the power price will be smaller with the increase in the number of outlets supplied by CSOs. This implies that the waiting time will be smaller compared with smaller waiting capacities, which will monotonically increase the optimal price. On the contrary, the power price will decrease with the decreasing waiting time, based on the number of outlets supplied by CSOs.

In order to evaluate how SGO $i$ makes decisions on the optimal pricing strategy with various initial prices, we get the simulation shown in Figure 6 through the proposed game-theoretical strategy. Figure 6 illustrates that the power price offered by SGO $i$ will be adjusted to a higher ideal value through several iteration steps when it is initially set as a smaller value, such as $p_{i}=5$ and $p_{i}=10$, respectively. On the contrary, the power price will converge to a smaller ideal value through several iteration steps when it is initially set as a higher value. From Figures 3-6, we see that it will converge to a stable value as long as the initial price is offered. 


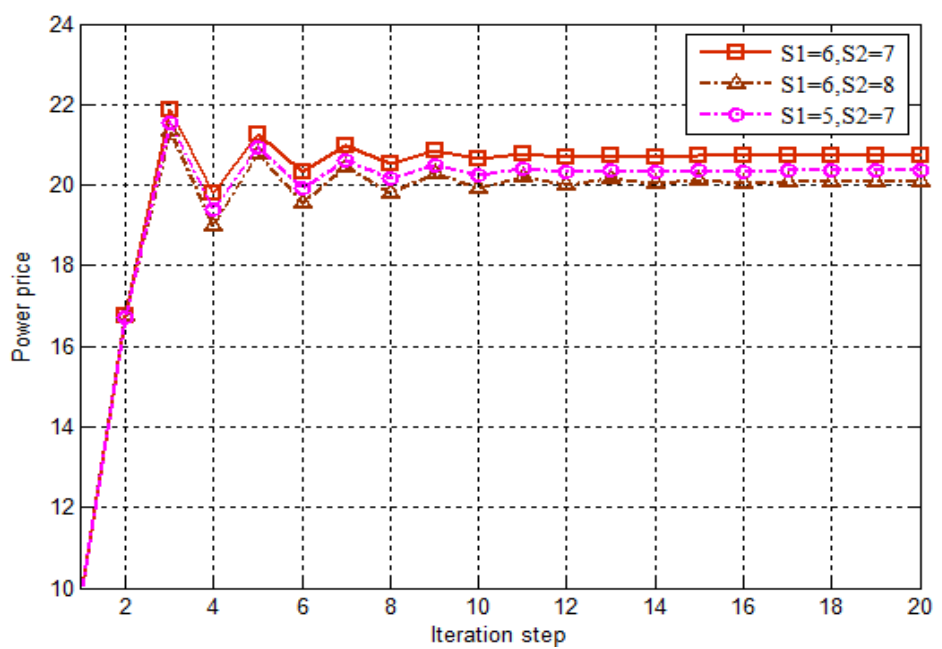

Figure 4. Power price offered by SGO $i$ versus the iteration step, in which its initial price $p_{i}=10 \$ / M W \cdot h$, $\eta_{1}=0.95, \eta_{2}=0.99$, and $C_{1}=C_{2}=3$, respectively.

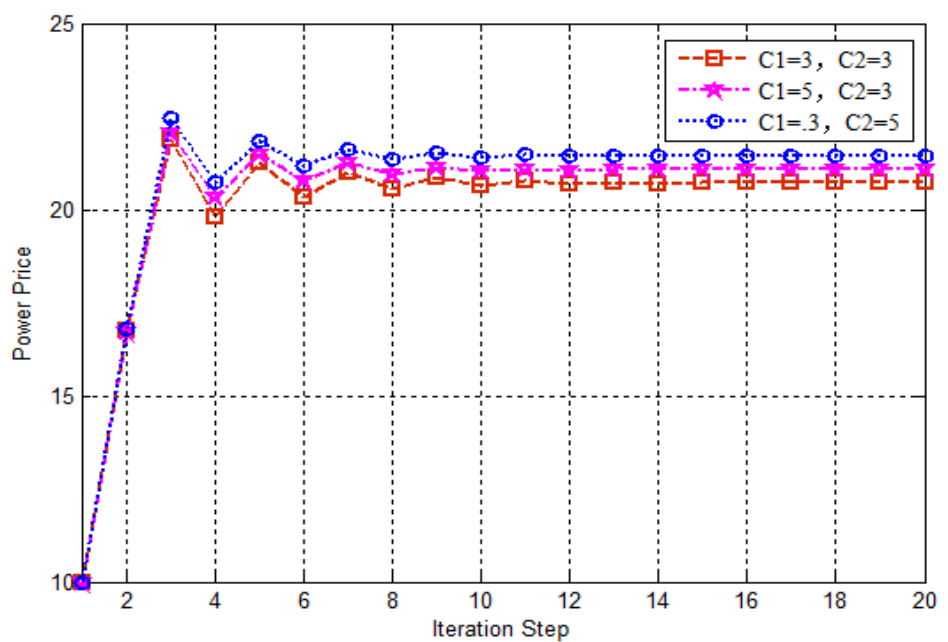

Figure 5. Power price offered by SGO $i$ versus the iteration step, in which its initial price $p_{i}=10 \$ / \mathrm{MW} \cdot \mathrm{h}$, $\eta_{1}=0.95, \eta_{2}=0.99$, and $S_{1}=6, S_{2}=7$, respectively.

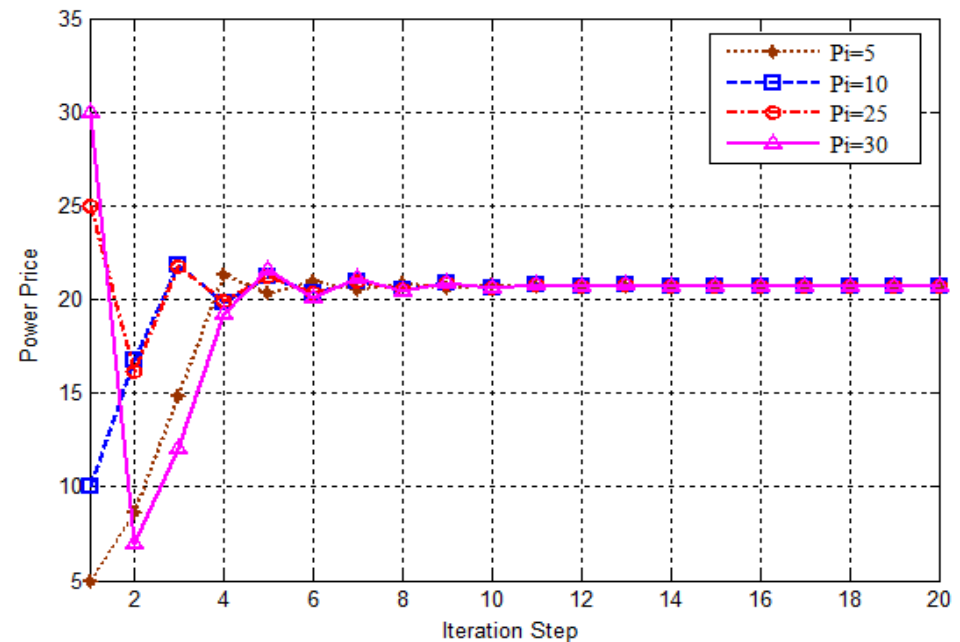

Figure 6. SGO's price compared with the iteration step, given $\eta_{1}=0.95, \eta_{2}=0.99$, and $C_{1}=C_{2}=3$, $S_{1}=6, S_{2}=7$. 
In addition, we investigate the optimal strategy for EV users to be charged based on the price given by CSOs. Following the price given by CSOs, Figure 7 demonstrates how EV users adjust their power demand to maximize their benefits. From Figure 7, we can observe that the value of power demand converges to the optimal value through several iteration steps, by using the proposed strategy. Meanwhile, we can also obtain that the amounts of power demand are different from each other. For example, power demand for EV users with $A_{k}=40$ is smaller than that with $A_{k}=50$, when all of them will be charged by CSO 1 . The results for EV users charged by CSO 2 is the same as the above. This implies that power demand for EV users with a higher satisfaction degree is larger than that with a lower satisfaction degree. From the simulation results in Figures 5 and 6, we can know that it matches the existence of SE for our proposed game-theoretical scheme in Section 5, through which it also can be obtained.

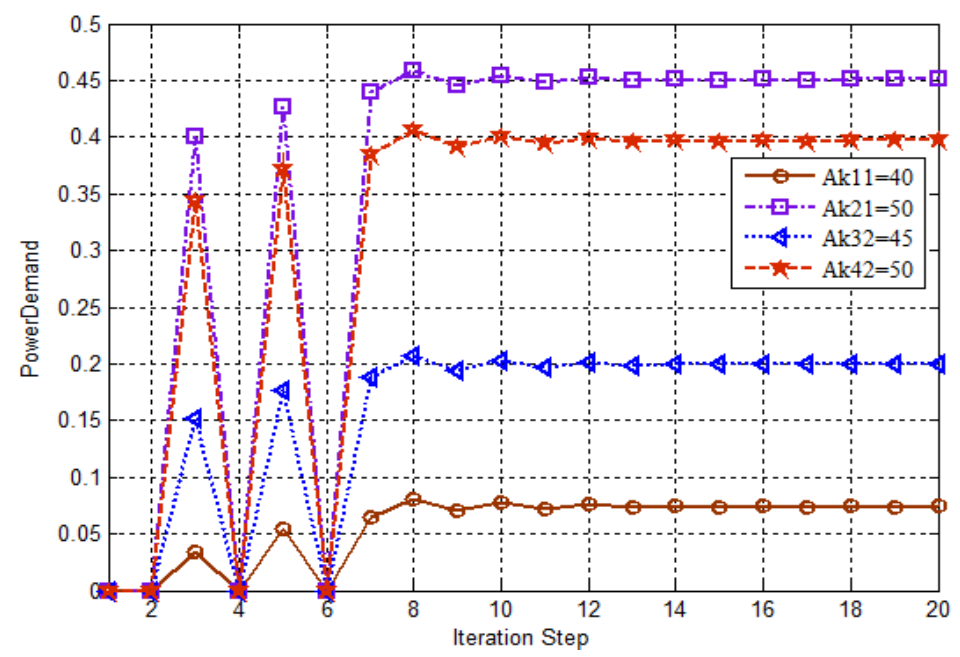

Figure 7. CSOs' power demand brought from CSOs compared with the iteration step, in which $\zeta_{1}=0.05$ and $\zeta_{2}=0.01$, and its initial price $p_{i}=10 \$ / \mathrm{MW} \cdot \mathrm{h}, W_{m 1}=0.3 \mathrm{~h}, W_{m 2}=0.4 \mathrm{~h}$, respectively.

From the results in Figures 2 and 3, we investigate the effect of BER and the outlets on the power price. This imposes that it can benefit both CSOs and EV users through adjusting the outlets and improving the performance of wireless communication. According to the simulation results in Figures 4-6, this means that both EV users and CSOs can also obtain the optimal solution, while a random initial power price offered by SGO is given.

Based on the above simulation results, we can know that our algorithm mainly focuses on the power supply in SGO. It also describes that the total utility of all parts in the charging system will be fixed, which cannot be affected with the increasing total power supply from SGO. This proves the existence and uniqueness of SE in our proposed game-theoretical scheme, which imposes the effectiveness of our proposal.

Finally, we compare the performance of the proposed optimal strategy with the existing scheme, with the same parameter setting in the simulations above. We adopt a uniform allocation scheme and a random allocation scheme to calculate the benefits of all EV users, respectively. Based on the utility function of EV users, we calculate the benefits of EV users with various total power demand supplied by SGO $i$, shown in Figure 8, using these three different methods. From Figure 8, we can observe that the benefits in the proposed scheme are higher than those in the other two schemes with increasing total power demand. This proves the effectiveness of our proposed algorithm in this paper. 


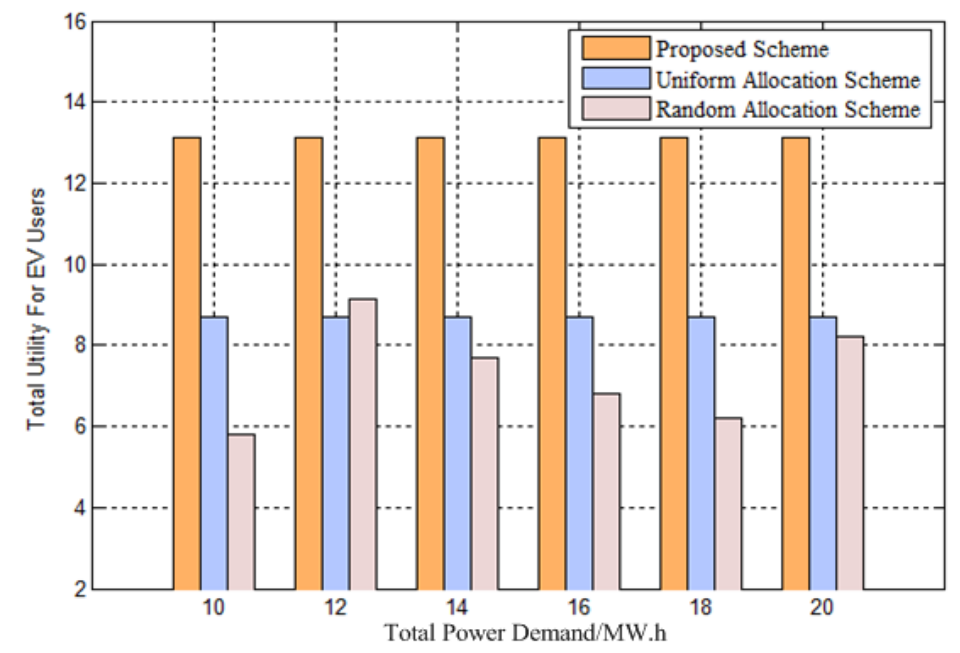

Figure 8. Total utility for EV users with different schemes, in which $\zeta_{1}=0.05$ and $\zeta_{2}=0.01$, and its initial price $P_{i}=10, W_{m 1}=0.3, W_{m 2}=0.4$, respectively.

In order to integrate the study in our paper with the practical application, it will be interesting to develop a smart power decision system (SPDS) to charging the service according to the limited power supply from SGO. The smart decision system is divided into two parts: (1) software: our proposed algorithm is designed as the major part of the optimal strategy, including the analysis of the data processing; (2) hardware: in order to satisfy many more EV users' power demand, the online information of EV users, CSOs, and SGOs is seen as the input of software in SPDS, respectively. At the same time, both the waiting time in CS and delay in Hetnet are taken into account. Further, it would be interesting to modify our proposed algorithm associated with the dynamic renewable power in the smart grid.

\section{Conclusions}

This work presents a game-theoretical approach to provide EVs with charging services supplied by CSOs, considering BER in Hetnet. A four-stage Stackelberg game scheme is developed to make optimal decisions for this charging system, composed of EV users, CSOs, and SGO. Considering the interaction among them, the utility function of each one is designed, while the load uncertainty is taken into account. Then, the SE can be obtained through the proposed iteration search algorithm, matching its existence and uniqueness in our proposed algorithm. Simulation results have been presented to demonstrate the performance of our proposal. For future work, it is interesting for us to extend our proposed algorithm with virtual technology, confronting realistic problems, i.e., the dynamic arrival of $\mathrm{EV}$ users and the delay of charging power in the queue.

Author Contributions: Conceptualization, S.C. and H.C.; Methodology, H.C.; Software, S.C.; Validation, S.C., H.C. and S.J.; Formal analysis, H.C.; Investigation, S.C.; Supervision, S.C.; Project administration, H.C.; Funding acquisition, S.J.

Funding: This research was funded by the National Natural Science Foundation of China under Grant No. 61572238 and the Key Project of Natural Science Research of the Anhui Provincial Department of Education under Grant No. KJ2016A431.

Acknowledgments: The author would like to thank the staff of Department of Computer Science and Technology, Tsinghua University, for their support during this study. Authors would like to thanks School of Physics and Electronic Engineering, Anqing Normal University for their generosity in financial assistance.

Conflicts of Interest: The authors declare no conflict of interest.

\section{References}

1. Bayram, I.S.; Michailidis, G.; Devetsikiotis, M.; Granelli, F. Electrical Power Allocation in a Network of Fast Charging Stations. IEEE J. Sel. Areas Commun. 2015, 31, 1235-1246. [CrossRef] 
2. Zhao, S.; Lin, X.; Chen, M. Robust Online Algorithms for Peaking-minimizing EV Charging under Multistage Uncertainty. IEEE Trans. Autom. Control 2017, 62, 5739-5754. [CrossRef]

3. Rakhymbay, A.; Khamitov, A.; Bagheri, M.; Alimkhanuly, B.; Lu, M.; Phung, T. Precise Analysis on Mutual Inductance Variation in Dynamic Wireless Charging of Electric Vehicle. Engergies 2018, 11, 624. [CrossRef]

4. Ma, T.; Wu, J.; Hao, L.; Yan, H.; Li, D. A Real-Time Pricing Scheme for Energy Management in Integrated Energy Systems a Stackelberg Game Approach. Engergies 2018, 11, 2858. [CrossRef]

5. De Hoog, J.; Alpcan, T.; Brazil, M.; Thomas, D.A.; Mareels, I. Optimal Charging of Electric Vehicles Taking Distribution Network Constraints into Account. IEEE Trans. Power Syst. 2015, 30, 365-375. [CrossRef]

6. Wang, M.; Liang, H.; Zhang, R.; Deng, R.; Shen, X. Mobility-aware Coordinated Charging for Electric Vehicles in VANET-enhanced Smart Grid. IEEE J. Sel. Areas Commun. 2014, 32, 1344-1360. [CrossRef]

7. Lee, W.; Schober, R.; Wonga, V. An Analysis of Price Competition in Heterogeneous Electric Vehicle Charging Stations. IEEE Trans. Smart Grid 2018. [CrossRef]

8. Sisinni, E.; Saifullah, A.; Han, S.; Jennehag, U.; Gidlund, M. Industrial Internet of Things: Challenges, Opportunities, and Directions. IEEE Trans. Ind. Inform. 2018, 14, 4724-4734. [CrossRef]

9. Xiong, H.; Xiang, T.; Zhu, Y.; Song, X.; Chen, H.; Chen, H. Electric Vehicle Public Charging Stations Location Optimal Planning. Autom. Electr. Power Syst. 2012, 36, 65-70.

10. Luan, T.; Shen, X.; Bai, F. Integrity-oriented content transmission in highway vehicular ad hoc networks. In Proceedings of the 2013 IEEE INFOCOM, Turin, Italy, 14-19 April 2013; Volume 12, pp. 2562-2570.

11. Cordeschi, N.; Amendola, D.; Baccarelli, E. Reliable Adaptive Resource Management for Cognitive Cloud Vehicular Networks. IEEE Trans. Veh. Technol. 2015, 64, 2528-2537. [CrossRef]

12. Jia, L.; Hu, Z.; Song, Y.; Luo, Z. Optimal Siting and Sizing of Electric Vehicle Charging Stations. In Proceedings of the 2012 IEEE International Electric Vehicle Conference, Greenville, SC, USA, 4-8 March 2012; pp. 1-6.

13. Liu, N.; Chen, Q.; Liu, J.; Lu, X.; Li, P.; Lei, J.; Zhang, J. A Heuristic Operation Strategy for Commercial Building Microgrids Containing EVs and PV System. IEEE Trans. Ind. Electron. 2014, 62, 2560-2570. [CrossRef]

14. Wang, F.; Han, L.; Wen, B. Substation Optimization Planning based on the Improved Orientation Strategy of Voronoi Diagram. In Proceedings of the 2nd International Conference on Information Science and Engineering, Hangzhou, China, 4-6 December 2010; pp. 1563-1566.

15. An, J.; Huang, B.; Kang, Q.; Zhou, M. PSO-based Method to Find Electric Vehicle's Optimal Charging Schedule under Dynamic Electricity Price. In Proceedings of the 2013 10th IEEE International Conference on Networking, Sensing and Control (ICNSC), Evry, France, 10-12 April 2013; pp. 913-918.

16. Mohsenian-Rad, H.; Ghamkhari, M. Optimal Charging of Electric Vehicles with Uncertain Departure Times: A Closed-form Solution. IEEE Trans. Smart Grid 2015, 6, 940-942. [CrossRef]

17. Lee, W.; Xiang, L.; Schober, R.; Wong, V.W. Electric Vehicle Charging Stations With Renewable Power Generators: A Game Theoretical Analysis. IEEE Trans. Smart Grid 2015, 6, 608-617. [CrossRef]

18. Guo, S.; Zhao, H. Optimal Site Section of Electric Vehicle Charging Station by Using Fuzzy TOPSIS based on Sustainability Perspective. Appl. Energy 2015, 158, 390-402. [CrossRef]

19. Yuan, W.; Huang, J.; Zhang, Y. Competitive Charging Station Pricing for Plug-in Electrical Vehicles. IEEE Trans. Smart Grid 2014, 8, 668-673.

20. Manshadi, S.D.; Khodayar, M.E.; Abdelghany, K.; Üster, H. Wireless Charging of Electric Vehicles in Electricity and Transportation Networks. IEEE Trans. Smart Grid 2017, 9, 4503-4512. [CrossRef]

21. Sun, Y.; Hu, X.; Liu, X.; He, X.; Wang, K. A Software-defined Green Framework for Hybrid EV-charging Networks. IEEE Commun. Mag. 2017, 55, 62-69. [CrossRef]

22. Yang, J.; Chou, L.; Chang, Y. Electric-vehicle Navigation System based on Power Consumption. IEEE Trans. Veh. Technol. 2015, 65, 5930-5943. [CrossRef]

23. Zhang, R.; Cheng, X.; Yangg, L. Flexible Energy Management Protocol for Cooperative EV-to-EV Charging. IEEE Trans. Intell. Transp. Syst. 2019, 20, 172-186. [CrossRef]

24. Hu, X.; Wang, K.; Liu, X.; Sun, Y.; Li, P.; Guo, S. Energy Management for EV Charging in Software-defined Green Vehicle-to-grid Network. IEEE Commun. Mag. 2018, 56, 156-163. [CrossRef]

25. Adan, I.; Resing, J. Queueing Theory; Eindhoven University of Technology: Eindhoven, The Netherlands, 2002.

26. Bayram, I.S.; Ismail, M.; Abdallah, M.; Qaraqe, K.; Serpedin, E. A Pricing-based Load Shifting Framework for EV Fast Charging Stations. In Proceedings of the 2014 IEEE International Conference on Smart Grid Communications (SmartGridComm), Venice, Italy, 3-6 November 2014; pp. 680-685. 
27. Karbasioun, M.M.; Lambadaris, I.; Shaikhet, G.; Kranakis, E. Optimal Charging Strategies for Electric Vehicles under Real Time Pricing. In Proceedings of the 2014 IEEE International Conference on Smart Grid Communications (SmartGridComm), Venice, Italy, 3-6 November 2014; pp. 746-751.

28. Jin, C.; Tang, J.; Ghosh, P. Optimal Electric Vehicle Charging with Energy Storage in Electricity Market. IEEE Trans. Smart Grid 2013, 4, 311-320. [CrossRef]

29. Ismail, M.; Bayram, I.; Abdallah, M.; Qaraqe, K. Optimal Planning of Fast Charging Facilities. In Proceedings of the 2015 First Workshop on Smart Grid and Renewable Energy (SGRE), Doha, Qatar, 22-23 March 2015; pp. 1-6.

30. Ovalle, A.; Hably, A.; Bacha, S. Optimal Management and Integration of Electric Vehicles to the Grid: Dynamic Programming and Game Theory Approach. In Proceedings of the 2015 IEEE International Conference on Industrial Technology (ICIT), Seville, Spain, 17-19 March 2015; pp. 2673-2679.

31. You, P.; Hsieh, Y. A Hybrid Heuristic Approach to the Problem of the Location of Vehicle Charging Station. Comput. Ind. Eng. 2014, 70, 195-204. [CrossRef]

32. Huang, S.; He, L.; Gu, Y.; Wood, K.; Benjaafar, S. Design of A Mobile Charging Service for Electric Vehicles in An Urban Environment. IEEE Trans. Intell. Transp. Syst. 2015, 16, 787-798. [CrossRef]

33. Lee, J.; Guo, J.; Choi, J.K.; Zukerman, M. Distributed Energy Trading in Microgrids: A Game Theoretic Model and Its Equilibrium Analysis. IEEE Trans. Ind. Electron. 2015, 62, 1-10. [CrossRef]

34. Molisch, A. Wireless Communications; Wiley: West Sussex, UK, 2005.

(c) 2019 by the authors. Licensee MDPI, Basel, Switzerland. This article is an open access article distributed under the terms and conditions of the Creative Commons Attribution (CC BY) license (http://creativecommons.org/licenses/by/4.0/). 